Conclusion TIMP3 directly induce apoptosis in mesenchymal cells, which is independent of death receptor ligands. Moreover, TIMP3-mediated induction of apoptosis involves the inhibition of MAPK/Erk1/2 as well as the Akt signalling pathway.

\title{
A45 INDUCTION OF DEATH RECEPTOR LIGAND-INDEPENDENT APOPTOSIS BY ENDOGENEOUS TIMP3 IN MESENCHYMAL CELLS
}

C Wunrau, D Wendholt, N Pundt, T Pap, B Dankbar University Hospital, Muenster, Germany

\subsection{6/ard.2010.129593g}

Introduction The matrix metalloproteinases (MMPs) and their endogenous regulators, the tissue inhibitors of MMPs (TIMPs 1-4) are responsible for the physiological remodelling of the extracellular matrix (ECM) by maintaining the balance between matrix destruction and formation. An imbalance between MMPs and TIMPs leads to excess MMP activity and is associated with ECM destruction in various inflammatory conditions such as rheumatoid arthritis and malignant tumours. Among all TIMPs, TIMP3 appears to play an outstanding role since, exclusively, TIMP3 has been shown to be able to induce apoptotic cell death. As little is known about the underlying mechanisms, we investigated the pro-apoptotic effect of recombinant and lentiviral overexpressed TIMP3 in the human mesenchymal cell line Cal78.

Methods Mesenchymal Cal78 cells were transduced with pLenti6/V5-D-TOPO-TIMP3 or the control vector pLenti6/ V5-GW/lacZ and a single lentiviral TIMP3-producing cell clone was generated. Induction of apoptosis was evaluated using the caspase 3/7 assay after transduction with or without administration of $100 \mathrm{ng} / \mathrm{ml}$ Fas ligand (FasL), tumour necrosis factor $\alpha$ (TNF $\alpha$ ), TNF-related apoptosis-inducing ligand (TRAIL) for 16h. Cell proliferation was assessed by bromodeoxyuridine incorporation. Analysis of mitogen-activated protein kinase (MAPK) activation was performed by western blot analysis using specific anti-phospho antibodies.

Results Lentiviral overexpression of TIMP3 in Cal78 cells led to a strong induction of apoptosis reflected by a fivefold increase in caspase 3 and 7 activity in the TIMP3-overexpressing cells compared with control (pLenti6/V5-GW/lacZ) or uninfected cells. Moreover, induction of apoptosis was further increased by TNFa, FasL and TRAIL. Notably, exogenous TIMP3 (recombinant hTIMP3, $2 \mu \mathrm{g} / \mathrm{ml}$ ) did not induce caspase 3 and 7 activity. Since TIMP3-producing cells can survive despite a high apoptosis rate, we analysed the proliferation rate of these cells and found a fourfold higher proliferation after lentiviral infection with TIMP3. Further detailed analysis showed that withdrawal of serum led to an upregulation of the TIMP3induced apoptosis, pointing to serum-derived survival factors. Furthermore, lentiviral overexpression of TIMP3 resulted in a decreased phosphorylation of cRaf, Erk1/2, p90RSK and Akt. 